\title{
Supporting Information for: \\ Photoelectron Spectroscopy of Benzene in the Liquid Phase and Dissolved in Liquid Ammonia
}

\author{
H. Christian Schewe* ${ }^{1, \text { a) }}$ Krystof Brezina, ${ }^{2,1}$ Vojtech Kostal, ${ }^{1}$ Philip E. Mason, ${ }^{1}$ Tillmann Buttersack, ${ }^{3}$ Dominik \\ M. Stemer, ${ }^{3}$ Robert Seidel, ${ }^{4}$ Wilson Quevedo, ${ }^{4}$ Florian Trinter, ${ }^{3,5}$ Bernd Winter, ${ }^{3}$ and Pavel Jungwirth*1, b) \\ ${ }^{1)}$ Institute of Organic Chemistry and Biochemistry of the Czech Academy of Sciences, Flemingovo nám. 2, \\ 16610 Prague 6, Czech Republic \\ ${ }^{2)}$ Charles University, Faculty of Mathematics and Physics, Ke Karlovu 3, 12116 Prague 2, \\ Czech Republic \\ ${ }^{3)}$ Molecular Physics, Fritz-Haber-Institut der Max-Planck-Gesellschaft, Faradayweg 4-6, D-14195 Berlin, \\ Germany \\ 4) Helmholtz-Zentrum Berlin für Materialien und Energie, Hahn-Meitner-Platz 1, D-14109 Berlin, \\ Germany \\ 5) Institut für Kernphysik, Goethe-Universität Frankfurt, Max-von-Laue-Str. 1, D-60438 Frankfurt am Main, \\ Germany
}

(Dated: 8 December 2021)

\section{S1. SOLUBILITY OF BENZENE OR OTHER AROMATIC SPECIES IN LIQUID AMMONIA}

As pointed out in the main manuscript a high enough concentration of neutral benzene or other aromatic species is a prerequisite to record spectral signatures with a good signal-to-noise ratio in photo-electron measurement with liquid ammonia microjets. Similarly to water, one can assume the solubility of the neutral benzene in liquid ammonia to be limited as well as it to be a function of temperature. The experimental measurement presented here should not be seen as a precise thermodynamic study but rather as an investigation to determine an order of magnitude which provides a good estimate for the upcoming work. Solubility measurements were carried out as follows: a $50 \mathrm{~mL}$ round-bottom flask was firstly evacuated and then filled with inert argon atmosphere before it was cooled down to $-78{ }^{\circ} \mathrm{C}$ using an ethanol bath with dry ice as coolant. Then, $\sim 10.1 \mathrm{~g}$ of liquid ammonia was condensed and then allowed to thermally equilibrate at $-78{ }^{\circ} \mathrm{C}$. We measured the temperature using a thermostat. Liquid benzene (Merck purity: $99.8 \%$ ) was added into the solution in portions of $0.1 \mathrm{~mL}$ using a syringe. It solidified instantaneously on the surface of the liquid ammonia due to its melting point of $5.5{ }^{\circ} \mathrm{C}$. It however dissolved within tens of seconds. The method of measurement was to add portions of $0.1 \mathrm{~mL}$ of liquid benzene, then to wait for the solution to warm up slowly to the point where only a transparent liquid solution and no solid benzene was visibly observable as white frozen benzene anymore. Here we noted the temperature $T$ and the concentration $c$. The thus acquired data-set is plotted as dots in Figure S1. The measured data were then fitted using a linear curve

\footnotetext{
a)Electronic mail: hanns_christian.schewe@uochb.cas.cz

b) Electronic mail: pavel.jungwirth@uochb.cas.cz
}

$c=a T+b$, while the parameters $a$ and $b$ where obtained using a least squares polynomial fit in NumPy library. Importantly, it is apparent that the solubility of benzene

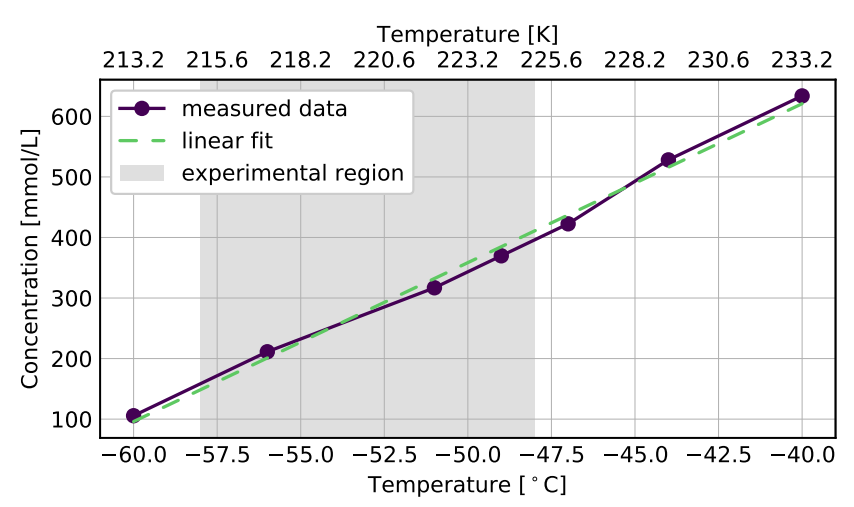

FIG. S1. Order of magnitude study of the solubility of benzene in liquid ammonia as a function of the temperature.

in liquid ammonia is an order of magnitude larger than in water throughout the temperature range presented here. Solubility of benzene shows a strong temperature dependence. These observations bring some implications for the upcoming work: firstly, the fact that the amount of dissolved benzene in liquid ammonia is on the order of $100 \mathrm{mmolL}^{-1}$ or higher is a very promising indication, that spectral signatures should be detectable in the XPS measurement. Secondly, the experiments with microjets of neat liquid ammonia showed that only a quite narrow temperature range between $210-225 \mathrm{~K}$ (around $-50{ }^{\circ} \mathrm{C}$ ) window is suitable in practice to produce stable liquid jets because either strong evaporative cooling close the boiling point causes the jets to freeze or clogging at low temperature near the melting point of ammonia. Figure S1 shows the temperature dependence of the solubility of benzene in liquid ammonia. It clearly illustrates that the solution needs to be thermally equilibrated within a very narrow window in order to avoid 
precipitation of solid benzene and clogging in the apparatus. This defines a strong technical boundary condition: the whole experimental setup has to be kept stable at a certain temperature as described in the experimental methodology section of the main manuscript.

\section{S2. CALIBRATION PROCEDURE OF ABSOLUTE BINDING ENERGIES}

As discussed in the experimental methodology section of the main manuscript the absolute binding energy of a particular spectral feature $\mathrm{BE}_{S F}$ can be determined by evaluating the energy difference between the kinetic energy of a spectral feature $\left(\mathrm{KE}_{S F}\right)$ and the kinetic energy value $\left(\mathrm{KE}_{c u t}\right)$ of the low kinetic energy curve at a given photon energy $h \nu$ following:

$$
\mathrm{BE}_{S F}=h \nu-\left(\mathrm{KE}_{S F}-\mathrm{KE}_{c u t}\right) .
$$

Upon application of a bias voltage $U$ all spectral features of the liquid-phase are shifted by the offset potential $\phi=e U$ where $e$ is the elementary charge. The gasphase peaks however do not shift but broaden once a bias voltage is applied. The higher the applied voltage the broader the gas-phase features become. Therefore, we chose a bias voltage of $\sim 40-50 \mathrm{~V}$ which ensures that the gas-phase features broaden to an extent such that they have become a quasi continuous offset. The remaining individual peak structure then corresponds to the liquid-phase spectral features as can be seen as black curve in the upper panel of Figure S2. A high-order (11th degree) polynomial (green curve) was fit to the data to account for the broadened gas-phase offset. The liquidonly VB spectral features are obtained by subtracting the high-order polynomial fit as can be seen as blue dots in the lower right panel of Figure S2. In order to determine
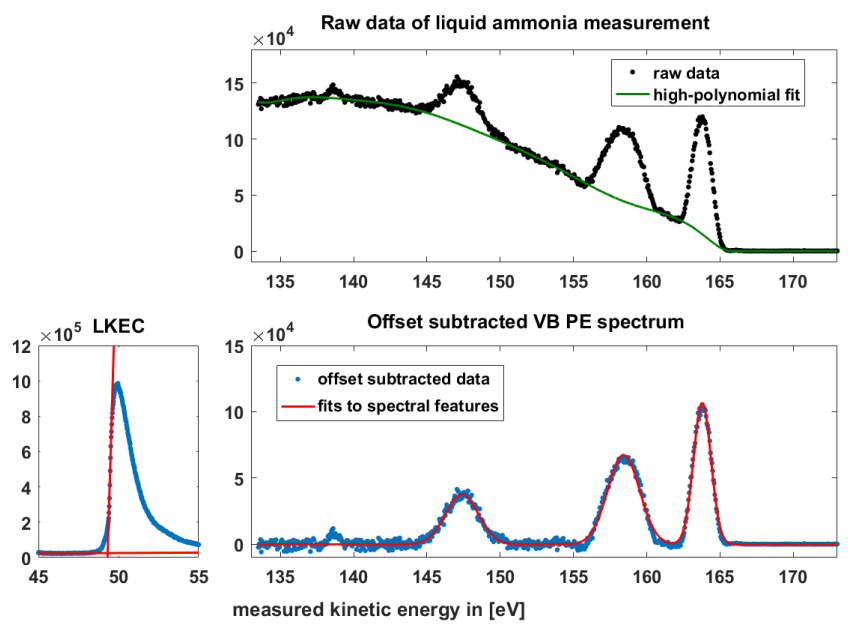

FIG. S2. PE spectrum of the low kinetic energy curve (left panel) and the valence band (right panel) of a $50 \mathrm{mM} \mathrm{NaI}$ liquid ammonia solution.

the absolute $\mathrm{BE}$ the liquid $\mathrm{HOMO}$ of the valence band is fit using a Gaussian function (red curve) - its centroid value determines $\mathrm{KE}_{S F}$. It should be noted that there is an ongoing discussion on how to determine the value of $\mathrm{KE}_{\text {cut }}$ : the experimental data of the low kinetic energy curve show a finite broadening - in sharp contrast to cutoff measurements of solid state samples - two different methods have been used in literature thus far: in the works from Olivieri et. al., Tissot et. al. or Ramirez et. al. a so-called secondary electron energy distribution curve (SEEDC) has been used which determines $\mathrm{KE}_{c u t}$ at the inflection point of the increasing slope of the low kinetic energy curve. ${ }^{\mathrm{S} 1-\mathrm{S} 3} \mathrm{In}$ the works of Thuermer et. al. and Malerz et. al. ${ }^{\mathrm{S} 4, \mathrm{~S} 5}$ the onset of the low kinetic energy curve is identified as the value of $\mathrm{KE}_{\text {cut }}$. Since the low kinetic energy curve shows a finite broadening, as can be observed in the left panel of Figure S2, the two methods of analysis will consequently lead to different values using the same experimental data. At this point too little is known about the broadening effects of the slope of the low kinetic energy curve to allow a more detailed analysis. In this work we follow the definition of the low kinetic energy curve that defines the smallest electron KE by which a PE can barely be ionized and by which it can just escape the solution. Hence, the onset of the low kinetic energy curve defines $\mathrm{KE}_{c u t}$ which is determined by the crossing point of the two fitted tangents which are shown in the left panel of Figure S2.

Calibration of the photon energy has been performed on a daily basis using the following procedure: Despite the fact that the photon energy was set by the monochromator of the beamline to be $h \nu=123.464 \mathrm{eV}$ also about $10 \%$ of $2^{\text {nd }}$ harmonic radiation of $h \nu=246.928 \mathrm{eV}$ passed through. On a daily basis we use this $2^{\text {nd }}$ harmonic radiation for the photon energy calibration for which we performed photo-electron yield measurements. We integrate the electron emission resulting from a so-called 2p3p3s Auger decay channel of an argon resonance at $h \nu=246.928 \mathrm{eV} .{ }^{\mathrm{S} 6}$ The notation $2 \mathrm{p} 3 \mathrm{p} 3 \mathrm{~s}$ resonant Auger process is used for example in Reference S7.

\section{S3. ESTIMATION OF THE SOLVATION ENERGY USING THE BORN EQUATION}

As pointed out in the main manuscript we approximate the gas-to-liquid shifts by estimating the Gibbs free energy of solvation is using the Born-Haber cycle ${ }^{\mathrm{S} 8}$ - see Figure 2 of the main manuscript. Additionally we use the Born equation to approximate the change in binding energy resulting from the change of the electronic polarizable continuum between the gas-phase and the liquidphase continuum:

$$
\Delta^{\mathrm{BH}} \mathrm{BE}_{\text {solute }}^{\text {solvent }}=\mathrm{E}^{\mathrm{solv}}=\frac{Z^{\prime 2} e^{2}}{8 \pi \epsilon_{0} R}\left(1-\frac{1}{n^{2}}\right),
$$

The high-frequency dielectric constant (square of refractive index $n) \epsilon_{r}=n^{2}$ of benzene and ammonia was taken 
as $1.50^{\mathrm{S} 9}$ and $1.39^{\mathrm{S} 10}$ respectively. To estimate the radius $R$ for the Equation S2 We used the interparticle distance given as the first maxima of radial distribution functions (RDF). Since the first peak of the radial distribution functions shows an asymmetry we not only deduce the distances at its maximum value but also the distances at half-width-half-maximum (HWHM). This also accounts for the spread in the average interparticle radius resulting from the fact that neither benzene nor ammonia are perfectly spherical entities. In ammonia the average nitrogen-nitrogen distance is 3.45 A with the HWHM distances being $[2.63 \AA, 4.03 \AA]^{\text {S11 }}$. Since ammonia is an almost spherical molecule, we estimate the radius $R$ to be half the interparticle distance which also approximates the excluded volume of an ammonia molecule best. Using Equation S2, we obtained for ammonia in liquid ammonia $\Delta^{\mathrm{BH}} \mathrm{BE}_{\mathrm{NH}_{3}}^{\mathrm{NH}_{3}(1)}=-2.01 \mathrm{eV}$ with the confidence interval being $[-1.72 \mathrm{eV},-2.64 \mathrm{eV}]$. For benzene in benzene we used the average center-of-mass to center-of-mass distance $5.9 \AA .{ }^{\mathrm{S} 12}$ Using equation $\mathrm{S} 2$ and $R=2.95 \AA$,

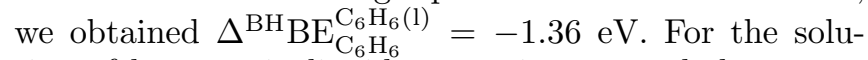
tion of benzene in liquid ammonia, we used the square of refractive index of ammonia (1.39). Values of the radius $R$ could be taken from Reference S13 where the excluded volume $R=2.7$ Ais derived from RDFs. Thus, $\Delta^{\mathrm{BH}} \mathrm{BE}_{\mathrm{C}_{6} \mathrm{H}_{6}}^{\mathrm{NH}_{3}(\mathrm{l})}=-1.29 \mathrm{eV}$ is calculated. For reasons of consistency with our argumentation - we use the same solute (benzene) in two different solvents (liquid benzene and liquid ammonia) - we use $R=2.95 \AA \AA$. ${ }^{\text {S12 }}$ Thus we determine $\Delta^{\mathrm{BH}} \mathrm{BE}_{\mathrm{C}_{6} \mathrm{H}_{6}}^{\mathrm{NH}_{3}(\mathrm{l})}=-1.18 \mathrm{eV}$.

The vaporization energy $\Delta E^{\text {vap }}$ per molecule is calculated from the standard enthalpy change of vaporization $\Delta H^{\text {vap }}$ minus the standard entropy change of vaporization, $T \Delta S^{\mathrm{vap}}$, where $\mathrm{T}=240 \mathrm{~K}$ is given in this system as the boiling temperature of ammonia. In the case of ammonia the values of $\Delta H^{\mathrm{vap}}=+23350 \mathrm{~J} / \mathrm{mol}$ and $T \Delta S^{\text {vap }}=97.41 \mathrm{~J} /(\mathrm{mol} \cdot \mathrm{K})^{\mathrm{S} 14}$ lead to the vaporization energy $\Delta E_{\mathrm{NH}_{3}}^{\mathrm{vap}}=0.04 \mathrm{eV}$. In the case of benzene $\Delta H^{\text {vap }}=+33900 \mathrm{~J} / \mathrm{mol}$ and $\Delta S^{\text {vap }}=114 \mathrm{~J} /(\mathrm{mol} \cdot \mathrm{K})^{\mathrm{S} 14}$ lead to the vaporization energy $\Delta E_{\mathrm{C}_{6} \mathrm{H}_{6}}^{\mathrm{vap}}=0.07 \mathrm{eV}$. Both vaporization energies are on the order of the error bar of the experiment and are therefore negligible within the approximations done to estimate the contributions for gas-to-liquid-phase shift.

\section{S4. SPECTRAL-INTENSITY ESTIMATION FOR BENZENE-AMMONIA SOLUTION}

The upper part of FigureS3 as well as Figure 7 of the main manuscript show the liquid-phase spectra of the pure benzene $\mathrm{I}_{\mathrm{C}_{6} \mathrm{H}_{6}}$ (red dots) and pure ammonia $\mathrm{I}_{\mathrm{NH}_{3}}$ (blue dots) solution which have been normalized such that the integrated area under the spectral features equals unity. The lower part shows a weighted sum of both pure spectra (green line) where $\mathrm{I}_{\mathrm{C}_{6} \mathrm{H}_{6}}$ has been scaled by a factor $\alpha$ before it is summed up with $\mathrm{I}_{\mathrm{NH}_{3}}$
- this allows to continuously manipulate the amount of the benzene contribution to the overall spectral intensity distribution.

To this end, the residuals of the spectrum of the weighted sum which has again been normalized and the normalized measured spectrum of $0.25 \mathrm{M}$ benzene in liquid ammonia have been calculated. Then the parameter $\epsilon$ is calculated from the sum of each residual squared. The area of the weighted sum spectrum has been found to equal the area of the measured spectrum of $0.25 \mathrm{M}$ benzene in liquid ammonia by optimizing the factor $\alpha$ by obtaining the smallest value of $\epsilon$. Thus, a ratio of the normalized spectral intensities of $\mathrm{I}_{\mathrm{C}_{6} \mathrm{H}_{6}} / \mathrm{I}_{\mathrm{NH}_{3}}=0.2 / 1$ has been found. In the following we estimate to what

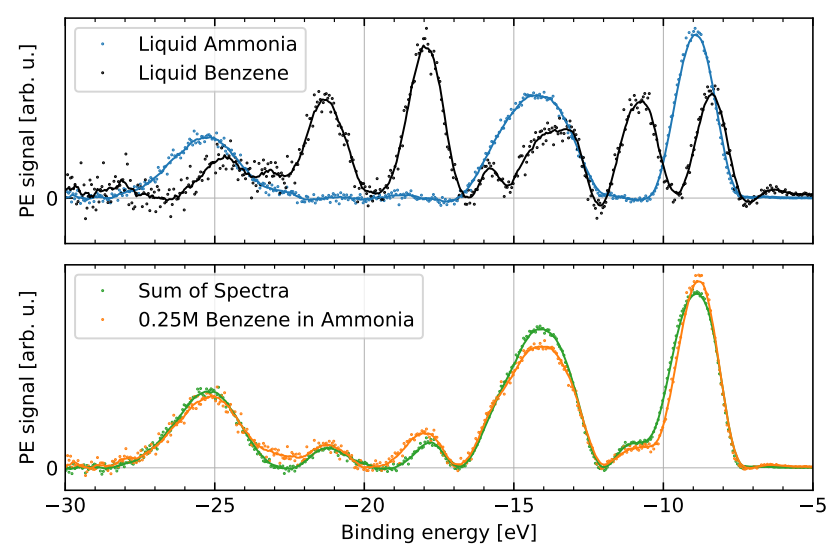

FIG. S3. Investigation of the gas-to-liquid-shift: details are given in the text.

extent the ratio of the particle numbers making up the solution can be compared to the ratio of the normalized spectral intensities taking details of the ionization process into account: 1.) The number of valence electrons is given by the atomic abundances within the two different molecules: the ratio of the number of $\mathrm{VB}$ electrons for benzene and ammonia is $30 / 8$. 2.) The number of particles per unit volume is $\mathrm{N}_{\mathrm{C}_{6} \mathrm{H}_{6}}=0.25 \mathrm{~mol} / \mathrm{l}$ and $\mathrm{N}_{\mathrm{NH}_{3}}=$ $41.1 \mathrm{~mol} / \mathrm{l}$ - thus follows the ratio: $0.25 / 41.1$ Multiplying these two ratios reflects the density of populated states (DoS) of the VB: $\operatorname{DoS}_{\mathrm{C}_{6} \mathrm{H}_{6}} / \mathrm{DoS}_{\mathrm{NH}_{3}} \sim 1 / 43.8$.

In order to be able to compare this ratio to the ratio of the spectral intensities $\left(\mathrm{I}_{\mathrm{C}_{6} \mathrm{H}_{6}} / \mathrm{I}_{\mathrm{NH}_{3}}\right)$ the ionization cross section $(\sigma)$ of the valence orbitals $\mathrm{C}_{2 \mathrm{P}}=0.09 \mathrm{Mbarn}$ and $\mathrm{N}_{2 \mathrm{P}}=0.29$ Mbarn given at $125 \mathrm{eV}^{\mathrm{S} 15}$ as well as the anisotropies related to experimental geometries $\beta_{\mathrm{C}_{6} \mathrm{H}_{6}}=$ 1.362 and $\beta_{\mathrm{NH}_{3}}=1.452$ are calculated. ${ }^{\mathrm{S} 15, \mathrm{~S} 16}$ The angular contribution is calculated from

$$
\left(1+\beta\left(\frac{1}{2}\left(3 \cos ^{2}(\varphi)-1\right)\right)\right)
$$

Since $\varphi=0$ in our experimental configuration, the weighted differential cross-sections are calculated using the formula:

$$
\frac{\sigma}{4 \pi}(1+\beta)
$$


Thus, the ratio accounting for differences in the ionization process for carbon atoms in benzene and nitrogen atoms ammonia states $0.0169 / 0.0566$, respectively.

Weighting this ratio with the derived ratio of the $\operatorname{DoS}_{\mathrm{C}_{6} \mathrm{H}_{6}} / \mathrm{DoS}_{\mathrm{NH}_{3}}$ estimates an spectral intensity distribution of $\mathrm{ESI}_{\mathrm{C}_{6} \mathrm{H}_{6}} / \mathrm{EsI}_{\mathrm{NH}_{3}} \sim 1 / 146.9 \times$ Thus, the ammonia peak areas should be on average be $\sim 147$ times larger than the benzene ones. The discrepancy between the ratio of $\sim 5$ observed in the measured PE spectra and the ratio of $\sim 147$ can be explained when assuming that benzene is surface active. Following this approximation: the concentration of benzene at the surface is $\sim 30$ times higher. Given the fact that Yeh and Lindau estimate the deviations in their calculations for the ionization cross sections and the anisotropies to be $\approx 20 \%^{\mathrm{S} 15}$ alone and given the fact we only do a rough estimate we can conclude that the concentration of benzene is enriched at the surface up to an order of magnitude.

\section{S5. GAS-TO-LIQUID SHIFT IN PURE AMMONIA REVISITED}

In Reference S17 the gas-to-liquid BE shift of the liquid HOMO $\left(3 \mathrm{a}_{1}\right)$ has been thoroughly investigated. The undisturbed gas-to-liquid-phase peak shift emerges by decreasing the photon flux which reduces the broadening or shifts induced by charging due to the photo-electron effect. The spectrum recorded at the lowest photon flux plotted in Figure S4 is shown in the supplementary information of Reference ${ }^{\mathrm{S} 17}$ only. The fitting of this spec-

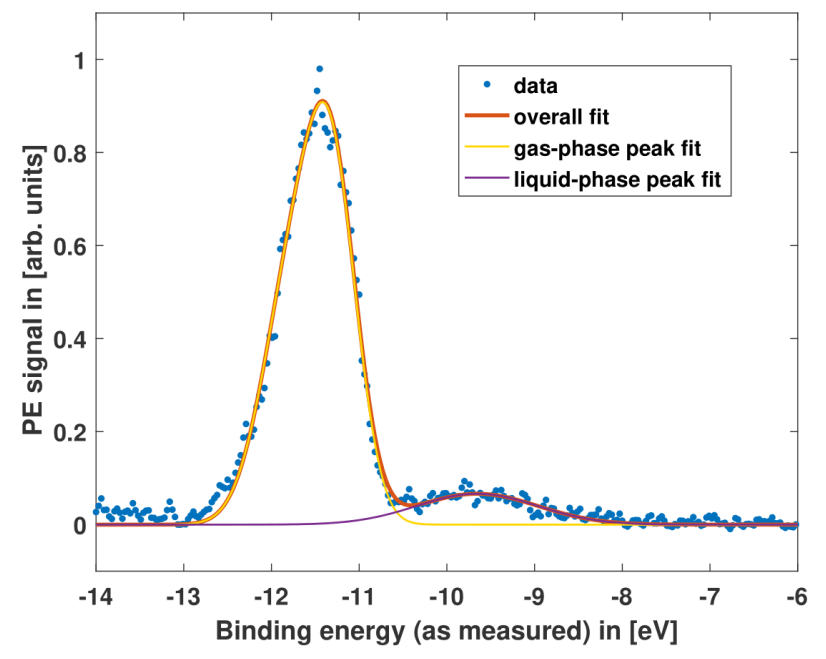

FIG. S4. Investigation of the gas-to-liquid-shift: details are given in the text.

trum is re-evaluated here to ensure that in this paper the same methodology is used throughout. The gas-phase peak (yellow line) has been fit using two Gaussian functions to account for its asymmetric peak shape, while the liquid-phase peak has been fit with a single Gaussian function (purple line). The overall fit (red line) fits the data (blue dots) very well which results in a gas-toliquid shift of $\Delta \mathrm{BE}_{\mathrm{NH}_{3}}=1.80 \mathrm{eV}$. The same procedure has been applied to determine the gas-to-liquid shift in benzene. Note, here the maximum of the gas-phase peak is used not its weighted mean.

Not only in Reference S17 but also in other investigations it is common practice to calibrate the liquid-phase $\mathrm{BE}$ of spectral features by measuring the gas-to-liquid shift of an individual spectral feature very accurately and referencing the gas-phase feature to a previously determined value. ${ }^{\text {S18 }}$ Spectral features of gas-phase molecules usually possess a vibrational progression which is, however, not always resolved due to a limited instrumental resolution. The individual peaks of the vibrational progression in ammonia is $\sim 0.11 \mathrm{eV}, \mathrm{S} 18$ while due to the limited spectral resolution of the setup $(\sim 0.25 \mathrm{eV})$ only a broad peak is recorded as can be observed in Figure S4.

Systematic errors can arise in this calibration method because it strongly relies on the assumption that the gas-phase peak shape is always the same and, therefore, its maximum can always be used to assign the vertical detachment energy (VDE). However, the population of the vibrational states is temperature dependent since in thermal equilibrium the Boltzmann distribution determines the population distribution of the vibrational states. While the vibrational progression in Reference S18 and hence the VDE stated therein has been determined under thermalized conditions at room temperature it can be doubted if the same conditions hold for ammonia molecules evaporating from a liquid ammonia jet under vacuum conditions. Not only do the molecules evaporate from a liquid jet at $\sim 220 \mathrm{~K}$ but also significant cooling of the vibrational degrees of freedom happens due to frequent inelastic collisions during the adiabatic expansion into vacuum. It has been shown that these dynamics lead to non-Maxwellian velocity distributions ${ }^{\mathrm{S} 19}$ and consequently also to non-thermal distributions of the vibrational states. Indications of a lower temperature can be observed when comparing the much larger asymmetry in gas-phase peak shape of Figure $\mathrm{S} 4$ relative to the one shown in Figure 10 and Figure 11 of Reference S18. Figure 4 of the main manuscript shows in its upper panel the gas phase spectrum of benzene. These spectra are in accordance with the spectra shown in Reference S20 where the vibrational state distribution shows a very asymmetric population distribution. In that experiment an effusive beam of gaseous benzene was used which results in a population distribution of the lowest vibrational levels. This indicates a temperature much lower than room temperature which can easily reach regimes between 10 $-100 \mathrm{~K}$.

It is obvious that the peak maximum shifts as a function of the underlying vibrational progression hence assigning the peak maximum can induce systematic errors. Since neither the temperature is known nor has the vibrational distribution of the ammonia gas been resolved 
no quantitative estimate on the value of the error can be given.

\section{S6. $\mathbf{G}_{0} \mathbf{W}_{0}$ VS. evGW COMPARISON FOR GAS-PHASE BENZENE}

In the discussion pertaining to Figure 4 of the main text, we claim that the current level of theory, the $\mathrm{G}_{0} \mathrm{~W}_{0}$ method building directly on DFT Kohn-Sham orbitals, does not reproduce certain features of the corresponding experimental spectrum. A straightforward way to improve on the insufficiencies of the current approximation would be to employ less aproximative methods that aim for single-particle states that guarantee self-consistency in the Green's function as well as the screened Coulomb interaction. Since optimized implementations of such methods are not yet routinely available for molecular systems, we tried to recalculate the EDOS over the same geometries with the evGW method: a simple generatization of the $\mathrm{G}_{0} \mathrm{~W}_{0}$ approach that does not introduce self-consistency in the Green's function, but rather performs a self-consistent cycle on the with the quasiparticle energy eigenvalues. As shown in Figure S5, the evGW indeed recovers the bimodality of the $3 \mathrm{e}_{2 g}$ and $1 \mathrm{a}_{2 u}$ peak at $-12 \mathrm{eV}$, but, at the same time, introduces a systematic shift of the spectrum to lower binding energies that destroys the good agreement of peak positions between the original EDOS and the experimental curve. Due to this reason, we consider the $\mathrm{G}_{0} \mathrm{~W}_{0}$ data superior and present them in the main text.

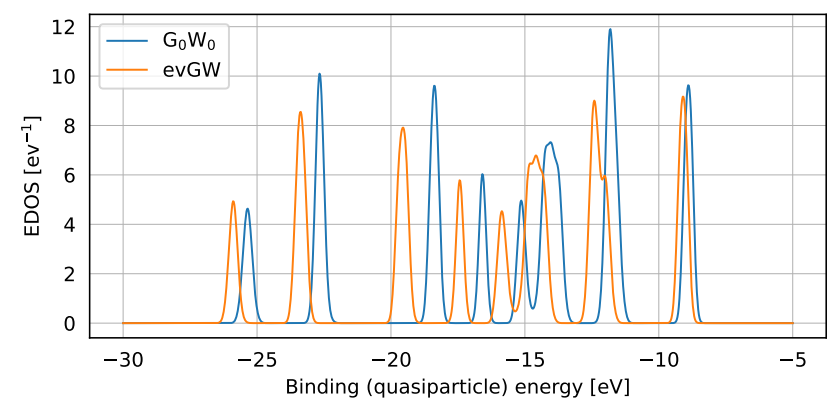

FIG. S5. Comparison of the gas-phase benzene GW EDOS calculated by the $\mathrm{G}_{0} \mathrm{~W}_{0}$ method (blue, identical to the data in Figure 4 of the main text) and calculated by the evGW method (orange).

\section{S7. ADDITIONAL RESULTS ON GAS-PHASE BENZENE ORBITAL ASSIGNMENT}

To aid the orbital assignment in the gas-phase benzene system, we verified that the double degeneracies predicted by the $e$-labels are consistent with the calculated EDOS. This was achieved by cumulative integration of the EDOS (Figure S6), relying on the fact that the density of states integrates to the number of one-particle electronic states (in this case, this corresponds to 30 explicitly represented electrons in the calculation). As such, the EDOS peaks are represented in the cumulative integral as step-wise increments by the number of orbitals contained in each peak. This conclusively identifies doubly degenerate peaks and, by comparison with the orbital labels, justifies the assignment.
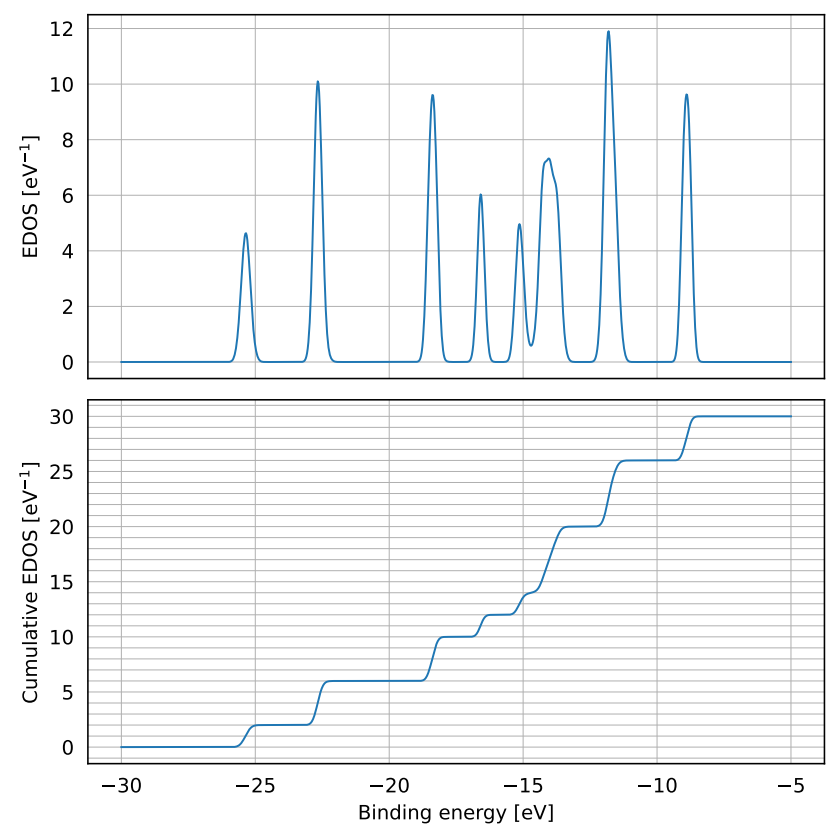

FIG. S6. Top panel: Gas-phase benzene EDOS. Bottom panel: cumulative integral of the gas-phase benzene EDOS.

\section{REFERENCES}

${ }^{\mathrm{S} 1}$ H. Tissot, J.-J. Gallet, F. Bournel, G. Olivieri, M. G. Silly, F. Sirotti, A. Boucly, and F. Rochet, "The Electronic Structure of Saturated $\mathrm{NaCl}$ and $\mathrm{NaI}$ Solutions in Contact with a Gold Substrate," Topics in Catalysis 2015 59:5 59, 605-620 (2015).

${ }^{\mathrm{S} 2}$ L. P. Ramírez, A. Boucly, F. Saudrais, F. Bournel, J.-J. Gallet, E. Maisonhaute, A. R. Milosavljević, C. Nicolas, and F. Rochet, "The Fermi level as an energy reference in liquid jet X-ray photoelectron spectroscopy studies of aqueous solutions," Physical Chemistry Chemical Physics 23, 16224-16233 (2021).

${ }^{\mathrm{S} 3} \mathrm{G}$. Olivieri, A. Goel, A. Kleibert, D. Cvetko, and M. A. Brown, "Quantitative ionization energies and work functions of aqueous solutions," Physical Chemistry Chemical Physics 18, 2950629515 (2016).

${ }^{\mathrm{S} 4}$ S. Thürmer, S. Malerz, F. Trinter, U. Hergenhahn, C. Lee, D. M. Neumark, G. Meijer, B. Winter, and I. Wilkinson, "Accurate vertical ionization energy and work function determinations of liquid water and aqueous solutions," Chemical Science 12, 10558-10582 (2021).

${ }^{\mathrm{S} 5}$ S. Malerz, F. Trinter, U. Hergenhahn, A. Ghrist, H. Ali, C. Nicolas, C.-M. Saak, C. Richter, S. Hartweg, L. Nahon, et al., "Lowenergy constraints on photoelectron spectra measured from liq- 
uid water and aqueous solutions," Physical Chemistry Chemical Physics 23, 8246-8260 (2021).

${ }^{\mathrm{S} 6}$ G. C. King, M. Tronc, F. H. Read, and R. C. Bradford, "An investigation of the structure near the L2,3 edges of argon, the M4,5 edges of krypton and the N4,5 edges of xenon, using electron impact with high resolution," Journal of Physics B: Atomic and Molecular Physics (1968-1987) 10, 2479 (1977).

${ }^{\mathrm{S} 7}$ S. Hüfner, Photoelectron Spectroscopy, Advanced Texts in Physics (Springer Berlin Heidelberg, Berlin, Heidelberg, 2003).

${ }^{S} 8$ M. Lundholm, H. Siegbahn, S. Holmberg, and M. Arbman, "Core electron spectroscopy of water solutions," Journal of Electron Spectroscopy and Related Phenomena 40, 163-180 (1986).

${ }^{S} 9$ K. Moutzouris, M. Papamichael, S. C. Betsis, I. Stavrakas, G. Hloupis, and D. Triantis, "Refractive, dispersive and thermooptic properties of twelve organic solvents in the visible and near-infrared," Applied Physics B 2013 116:3 116, 617-622 (2013).

S10 John R. Rumble, CRC Handbook of Chemistry and Physics, 101st ed., edited by John R. Rumble (CRC Press Taylor \& Francis, Boca Raton, FL, 2020).

${ }^{S 11}$ Private communication with Tomas M. and Ondrej M.

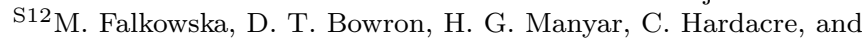
T. G. A. Youngs, "Neutron Scattering of Aromatic and Aliphatic Liquids," ChemPhysChem 17, 2043-2055 (2016).

${ }^{\mathrm{S} 13}$ K. Brezina, P. Jungwirth, and O. Marsalek, "Benzene Radical Anion in the Context of the Birch Reduction: When Solvation Is the Key," Journal of Physical Chemistry Letters 11, 6032-6038 (2020).

S14 J. Donald R. Burgess, "Thermochemical Data," in NIST Chemistry WebBook, NIST Standard Reference Database Number 69
(National Institute of Standards and Technology, 2018) Chap. Ed. by P.J.

${ }^{S 15}$ J. J. Yeh and I. Lindau, "Atomic subshell photoionization cross sections and asymmetry parameters: $1 \leq \mathrm{Z} \leq 103$," Atomic Data and Nuclear Data Tables 32, 1-155 (1985).

${ }^{S} 16$ D. E. Parry, "Atomic calculation of photoionization crosssections and asymmetry parameters," Rapid Communications in Mass Spectrometry 8, 579 (1994).

S17T. Buttersack, P. E. Mason, R. S. McMullen, T. Martinek, K. Brezina, D. Hein, H. Ali, C. Kolbeck, C. Schewe, S. Malerz, et al., "Valence and Core-Level X-ray Photoelectron Spectroscopy of a Liquid Ammonia Microjet," Journal of the American Chemical Society 141, 1838-1841 (2019).

${ }^{S 18}$ D. Edvardsson, P. Baltzer, L. Karlsson, B. Wannberg, D. M. P. Holland, D. A. Shaw, and E. E. Rennie, "A photoabsorption, photodissociation and photoelectron spectroscopy study of $\mathrm{NH}_{3}$ and $\mathrm{ND}_{3}$," Journal of Physics B: Atomic, Molecular and Optical Physics 32, 2583 (1999).

${ }^{\mathrm{S}} 19 \mathrm{M}$. Faubel and T. Kisters, "Non-equilibrium molecular evaporation of carboxylic acid dimers," Nature 1989 339:6225 339, 527-529 (1989).

${ }^{\text {S20 }}$ P. Baltzer, L. Karlsson, B. Wannberg, G. Öhrwall, D. M. Holland, M. A. MacDonald, M. A. Hayes, and W. Von Niessen, "An experimental and theoretical study of the valence shell photoelectron spectrum of the benzene molecule," Chemical Physics 224, 95-119 (1997). 